\title{
Radiation dose-response (a Bayesian model) in the radiotherapy of the localized prostatic adenocarcinoma: the reliability of PSA slope changes as a response surrogate endpoint
}

\author{
Reza Ali Mohammadpour ${ }^{1}$, Jamshid Yazdani- Charati ${ }^{1}$, SZahra Faghani ${ }^{1}$, Ahad Alizadeh ${ }^{2}$, Mohammadreza \\ Barzegartahamtan ${ }^{\text {Corresp. } 3}$ \\ 1 Department of Biostatistics, Faculty of Health, Mazandaran University of Medical Sciences, Sari, Iran \\ 2 2. Department of Epidemiology and Reproductive Health, Reproductive Epidemiology Research Center, Royan Institute for Reproductive Biomedicine, \\ ACECR, Tehran, Iran \\ 3 Department of Radiation Oncology, Firoozgar Hospital, Iran University of Medical Sciences, Tehran, Iran \\ Corresponding Author: Mohammadreza Barzegartahamtan \\ Email address: Barzegartahamtan.mr@iums.ac.ir
}

Purpose: One of the characteristics of Prostate-Specific Antigen (PSA) is PSA slope. It is the rate of diminishing PSA marker over time after radiotherapy (RT) in prostate cancer (PC) patients. The purpose of this study was to evaluate the relationship between increasing RT doses and PSA slope as a potential surrogate for PC recurrence. Patients and Methods: This retrospective study was conducted on PC patients who were treated by radiotherapy in the Cancer Institute of Iran during 2007-2012. By reviewing the records of these patients, the baseline PSA measurement before treatment (iPSA), Gleason score (GS), clinical T stage (T. stage), and periodic PSA measurements after RT and the total radiation dose received were extracted for each patient separately. We used a Bayesian dose-response model, analysis of variance, Kruskal-Wallis test, Kaplan-Meier product-limit method were used for analysis. Probability values less 0.05 were considered statistically significant. Results: Based on the D'Amico risk assessment system, 13.34\% of patients were classified as "Low Risk", 51.79\% were "Intermediate Risk", and 34.87\% were "High Risk". In terms of radiation doses, $12.31 \%$ of the patients received fewer than $50 \mathrm{~Gy}$, $15.38 \%$ received 50 to $69 \mathrm{~Gy}, 61.03 \%$ received $70 \mathrm{~Gy}$, and $11.28 \%$ received more than 70 Gy. The PSA values decreased after RT for all dose levels. The slope of PSA changes was negative for 176 of 195 patients. By increasing the dosage of radiation, the PSA decreased but these changes were not statistically significant $(p=0.701)$ and PSA slope as a surrogate end point cannot met the Prentice's criteria for PC recurrence. Conclusion: Significant changes in the dose-response relationship were not observed when the PSA slope was considered as the response criterion. Therefore, although the absolute value of the PSA decreased with increasing doses of RT, the relationship between PSA slope changes and 
increasing doses was not clear and cannot be used as a reliable response surrogate endpoint. 
1 Radiation dose-response (a Bayesian model) in the radiotherapy of the localized prostatic

2 adenocarcinoma: the reliability of PSA slope changes as a response surrogate endpoint

5 Running title: PSA slope after radiotherapy of prostate cancer

Authors: Reza Ali Mohammadpour ${ }^{1}$, Jamshid Yazdani- Charati ${ }^{1}$, SZahra Faghani ${ }^{1}$, Ahad Alizadeh ${ }^{2}$, 10 Mohammadreza Barzegartahamtan ${ }^{3 *}$

*Corresponding author: Department of Radiation Oncology, Firoozgar Hospital, Iran 


\section{Abstract}

Purpose: One of the characteristics of Prostate-Specific Antigen (PSA) is PSA slope. It is the rate of PSA change over time after radiotherapy (RT) in prostate cancer (PC) patients. The purpose of this study was to evaluate the relationship between increasing RT doses and PSA slope as a potential surrogate for PC recurrence.

Patients and Methods: This retrospective study was conducted on PC patients who were treated by radiotherapy in the Cancer Institute of Iran from 2007 to 2012. By reviewing the records of these patients, the baseline PSA measurement before treatment (iPSA), Gleason score (GS), clinical T stage (T. stage), and periodic PSA measurements after RT and the total radiation dose received were extracted for each patient separately. A Bayesian dose-response model, analysis of variance, Kruskal-Wallis test, Kaplan-Meier product-limit method were used for analysis. Probability values less 0.05 were considered statistically significant.

Results: Based on the D'Amico risk assessment system, 13.34\% of patients were classified as "Low Risk", 51.79\% were "Intermediate Risk", and 34.87\% were "High Risk". In terms of radiation doses, $12.31 \%$ of the patients received fewer than $50 \mathrm{~Gy}, 15.38 \%$ received dosage in interval [50 70) Gy, 61.03\% received $70 \mathrm{~Gy}$, and $11.28 \%$ received more than $70 \mathrm{~Gy}$. The PSA values decreased after RT for all dose levels. The slope of PSA changes was negative for 176 of 195 patients. By increasing the dosage of radiation, the PSA decreased but these changes were not statistically significant $(\mathrm{p}=0.701)$ and PSA slope as a surrogate end point cannot met the Prentice's criteria for PC recurrence.

Conclusion: Significant changes in the dose-response relationship were not observed when the PSA slope was considered as the response criterion. Therefore, although the absolute value of the PSA decreased with increasing doses of RT, the relationship between PSA slope changes and increasing doses was not clear and cannot be used as a reliable response surrogate endpoint. 
86

87

\section{INTRODUCTION}

Prostate cancer is an important health problem in men (Esfahani, Ataei \& Panjehpour 2014, and Wilt \& Ahmed 2013). Also, it is reported as the most common malignancy and the second leading cause of cancer related death in men in many parts of the world (Bidgoli, Jabari \& Zavarhei 2013, and Obort, Ajadi \& Akinloye 2013). Radiotherapy plays an essential role in the treatment of prostate cancer patients. Both the PSA amount and its changes over time, including velocity, density, and doubling time, are important in assessing response to RT (D'amico et al. 2003, Molenberghs et al. 2002). Some of PSA metrics (e.g. PSA velocity greater than 1.5 ng/ml/yr and PSA doubling time $<6$ months) are surrogate for PC mortality or overall survival (OS) (D’amico 2005) but others (e.g. PSA decline $>=30 \%$ and PSA doubling time $>12$ months) are not surrogate endpoint. (Collette 2008, Halabi et al 2013 and Valicenti et al 2006). Another of these changes over time is the PSA slope. In fact, it is the rate of PSA change over time after RT and has been previously discussed in the literature (Vollmer \& Montana 1999, Suzman, Zhou, Zahurak, Lin \& Antonarakis 2015, Bellera, Hanley, Joseph \& Albertsen 2008). Biochemical failure (BF) has various definitions such as two or three PSA rises, post-nadir increase to $>=3$ $\mathrm{ng} / \mathrm{ml}$ above the nadir and PSA value to be greater than a fixed cutoff level. Rising PSA was considered in these definitions (Takamiya et al 2003) but PSA slope was used as a continuous variable or categorized outcome for determining disease-free survival in a few studies. ProustLima et al (2008) assessed the relationship between prognostic factors, PSA dynamics and clinical failure (CF) using a complex two-stage model. They used a linear mixed model for prediction of PSA evolution in three phases after RT. Takamiya et al 2003 found that a zero PSA slope in post treatment PSA supports cure of patients with long-term follow up after RT, but there are few studies that demonstrated the surrogacy of PSA slope for CF end point.

Some studies have suggested that biochemical responses such as freedom from BF would improve with increasing doses, but the freedom from CF or OS of patients did not change significantly (Budäus et al. 2012, Wolff et al. 2009, Al-Mamgani et al. 2008, Peeters et al. 2006). Also dose escalation advantages and dose effect for low, intermediate and high risk groups were reported different in literature (Pollack et al. 2002, Kuban et al. 2008, Zietman et al. 2005) and there is controversy about the dose escalation benefits in the various risk groups especially low risk patients. Further research in this field will probably clarify these differences. Also it is important to analyze the PC clinical relapse (as a true endpoint) and a within-subject response PSA slope (as a potential surrogate endpoint) of dose-escalated therapy (>70 Gy) in a populationbased cohort. 
119 Some studies have shown that radiation dose escalation is related to the risk of recurrence, 120 treatment response and OS by using a joint modeling approach (Taylor \& Wang 2002, Prentice 121 RL 1989). However, the impact of radiation dose escalation on PSA slope is not clear. Another 122 study (Alizadeh, Mohammadpour \& Barzegar 2013) showed that the PSA slope was related to 123 the recurrence onset time. Different statistical methods such as mixture model, exponential model 124 and Baysian model are used to investigate the relationship between dose and response in 125 medicine. The dose-response Bayesian model tries to identify a connection between the average 126 responses at nearby doses. We consider the PSA slope as a treatment response and estimate the 127 posterior mean. Working within a Bayesian framework avoids many of the implicit assumptions 128 such as small sample size that restrict the validity of classical likelihood methods. For instance, 129 most data sets used for dose-response analysis are very small, containing only a few dose groups 130 with a few exposed subjects. In these situations, in spite of complexity in Bayesian model, 131 Markov Chain Mont Carlo (MCMC) sampling method is quite effective at handling complex models and provide a clear advantage over Maximum-Likelihood estimation (MLE) (Hamra, MacLehose \& Richardson 2013 and Leninger 2009). The present study investigates the impact of radiation dose escalation on the PSA slope after radiotherapy by using a statistical Bayesian model. If the result of this study indicates that dose is a worthy predictor of PSA slope, then Prentice’s criteria (Prentice 1989 and Heller 2015) for PSA slope surrogate for the recurrence time of PC are evaluated.

\section{MATERIALS AND METHODS}

\section{Patients}

140 This historical cohort study was performed after getting approval from the ethical committee of Mazandaran University of Medical Sciences (IR.MAZUMS.REC.1394.1347). For this study, all

142 localized prostate cancer patients who were treated by RT in the Cancer Institute of Iran (Tehran) 143 from 2007 to 2012, were investigated retrospectively. The study data was taken from existing 144 files of previously treated patients and the subject of consent form was not applicable 145 (ProstateCancerDataS1, PSArepeatedMeasurementsS2).

146 By reviewing the records of these patients, the baseline PSA measurement before treatment 147 (iPSA), Gleason score (GS), clinical T stage (T. stage), and periodic PSA measurements after RT 148 and the total dose of radiation received were extracted for each patient separately. All the patients 149 were classified into risk assessment groups. The stratification of patients was done with the use of D’Amico system as follows: patients with all conditions $i P S A \leq 10 \wedge G S \leq 6 \wedge T$. stage $=T 1-T 2 a$ 
152 Intermediate-Risk, and with a condition $P S A>20 \vee G S=8-10 \vee T$. stage $\geq T 2 c-T 3$ into High-

153 Risk prostate cancer (Rodrigues et al. 2012 and D'Amico et al. 1998). Also, all patients were 154 divided into four groups in terms of the total dose received as the main variable ( $<50$ Gy, [50 70)

155 Gy, =70 Gy and 70< Gy). After obtaining the pre-treatment and post-treatment values, the slope 156 of PSA changes was calculated for each patient. In this study, PSA slope is the slope of the linear 157 regression of PSA repeated measurements vs. time and was calculated by slope function in Excel 158 software. Finally, the relationship between the radiation dose received and the slope of PSA 159 changes was investigated.

160 Statistical method: In this study, we used a Bayesian dose-response model for analysis. In this 161 model, the slope of PSA changes was calculated and considered as treatment response of the $\mathrm{j}^{\text {th }}$ 162 individual at the $\mathrm{i}^{\text {th }}$ dosage level as follows:

163 slope $_{i j} \operatorname{Normal}\left(\mu_{i}, \sigma_{\text {slope }}^{2}\right)$

$164 \mu_{i}=$ the mean response for the $i^{t h}$ dosage level, and $\sigma_{\text {slope }}^{2}=$ the common variation at all dose levels.

165 Prior distributions:

$166 d_{i}=$ square root of the distance between the current and previous dosage levels,

$167 \sigma_{\mu}^{2}=$ the priori variance of the mean response at each dosage level (Leninger 2009).

$168 \mu_{i} \operatorname{Normal}\left(\mu_{i-1}, d_{i} \sigma_{\mu}^{2}\right)$

$169 \sigma_{\text {slope }}^{2}$ Gamma

$170 \quad \sigma_{\mu}^{2}$ Gamma

$171 \mu_{0} \operatorname{Normal}(0,0.0001)$

172 Statistical software package that uses Markov Chain Monte Carlo, BOA package of R software 173 (available from http://CRAN.R-project.org/.) (Smith 2007) trace plot, boxplot and ANOVA-type 174 diagnostic test were used for analysis and interpretation of the above mentioned data. Written R 175 code was explained in the supplemental file (RcodesBOAlinksS3).

176 The method of analysis of variance was used to compare the mean values of continuous 177 variables, and the non-parametric Kruskal-Wallis test was used to compare the median values of 178 PSA slope among the dose levels. The Kaplan-Meier product-limit method was used to estimate 179 probabilities of time to recurrence or survival time, both measured from the start of RT. The log180 rank test was used to compare distributions. Probability values less 0.05 were considered 181 statistically significant. 


\section{RESULTS}

184

185

186

187

188

189

190

191

192

193

194

195

196

197

198

199

200

201

202

203

204

205

206

207

208

209

210

211

212

213

214

215

In this study, 195 localized prostate cancer patients who were treated by RT in the Cancer Institute of Iran between 2007 and 2012 were identified. The shortest and longest durations of follow-up were four and 67 months, respectively. The mean follow-up duration was 19.60 months. In terms of risk grouping, $13.34 \%$ of the patients were classified as "Low Risk", $51.79 \%$ were "Intermediate Risk", and 34.87 \% were "High Risk". The slope of PSA changes was negative for 176 patients, meaning that for most of the patients, the overall trend of PSA trajectory was descending. Table 1 shows the frequency distribution of patients in each radiation dose received level both in non-stratified and stratified patients. The PSA slope after RT by the follow-up period is illustrated in Figure 1. The results of this study indicated that the long interval from the baseline PSA before RT to end of follow-up time was not correlated with a lower slope ( $p=0.98$ ). The maximum number of PSA measurements for patients after RT was 19 and correlation between follow-up duration and number of PSA measurements was statistically significant $(\mathrm{r}=0.77, \mathrm{p}<0.01)$.

Table 2 lists the posterior parameters, including posterior mean, posterior median, posterior standard deviation, and posterior credible interval (2.5\% and $97.5 \%$ percentiles), based on 12000 iterations in Post RT patients in a regression normal model.

Figure 2 (A-D) is a boxplot that compares the posterior mean parameter of PSA slope in the dose levels and risk groups. The boxplots are arranged in ascending order. This figure demonstrated that PSA decreased after RT for all doses. When the radiation doses were increased, the post-RT PSA slope dropped further. The greatest reduction in the PSA slope was recorded for doses greater than $70 \mathrm{~Gy}$. By increasing the dosage of radiation, PSA is decreasing at a faster rate, but these changes were not statistically significant and the dose response curve became a straight line for the total PSA slope mean in the all doses levels.

\section{The surrogacy testing by the Prentice's criteria:}

The Prentice criteria are the following. (I) Treatment is predictor for true endpoint. (II) Treatment is predictor for surrogate endpoint. (III) Surrogate is correlated with true endpoint. (IV) The full effect of the treatment on true endpoint is explained by surrogate endpoint.

Dose level was a statistically significant predictor of disease-free survival (Figure 3-A) and was satisfied in first condition of surrogacy. Mann-Whitney Test for total dose by recurrence event shows that the mean-ranks of doses is different in two groups with or without recurrence $(\mathrm{p}<0.001)$. 
216 As table 2 indicates, the Bayesian credible interval among 4 dose groups is overlapped and 217 median of PSA slope in groups is same. There was no significant difference among four groups 218 in PSA slope mean $(\mathrm{p}=0.705)$. Also the distributions of slope is the same across categories of 219 total dose by Kruskal-Wallis Test $(\mathrm{p}=0.902)$. Consequently total dose was not a worthy 220 prognosticator for PSA slope as a continuous outcome, because of this the Prentice's second 221 criterion was not met.

222 PSA slope was a statistically significant predictor of time to PC recurrence (disease-free 223 survival). The means of disease-free survival time in patients who had positive and no positive 224 PSA slope values were estimated 33.35 (CI 95\% 19.2, 47.4) vs. 59.6 (CI 95\% 52.8, 66.4) months 225 respectively by using the Kaplan-Meier method (log rank test $\mathrm{p}<0.001$ ). Figure 3-B demonstrates 226 the effect of changes in PSA slope on disease-free survival using Kaplan-Meier analysis. The 227 change in PSA slope (as a dichotomized variable) was significantly prognostic for disease-free survival (log rank test, $\mathrm{p}<0.001$ ) therefore the third criterion of Prentice was met. But MannWhitney Test for slope by recurrence events shows that the mean-ranks of slope is not different in two groups with or without PC recurrence $(\mathrm{p}=0.345)$. Consequently PSA slope as a continuous outcome did not meet the third criterion of Prentice.

In a multivariate logistic model, with PSA slope and dose level as inputs to the model, the dose is remained statistically significant $(\mathrm{p}=0.007)$, but the PSA slope was not significantly related to recurrence $(\mathrm{p}=0.594)$. Also figure $4(\mathrm{~A}, \mathrm{~B})$ demonstrates the effect of total dose on disease-free survival when PSA slope values are zero or less (no positive) vs. positive PSA slope group by using Kaplan-Meier analysis. The change in radiation total dose was significantly prognostic for disease-free survival in the first group (log rank test, $\mathrm{p}<0.001)$ but not in the second group (log rank test, $\mathrm{p}=0.9$ ). This result indicates that patients with negative or zero PSA slope had significantly PC recurrence less than others if they had been previously treated with higher doses rather than low doses. Thus PSA slope as a surrogate endpoint cannot fully explain the effect of dose and the Prentice's criterion 4 was not met for capturing total effects of radiation dose by PSA slope on PC recurrence.

\section{DISCUSSION:}

244 The prostate-specific antigen is a biomarker and its changes can be used as a surrogate endpoint 245 for response evaluation in prostate cancer patients (D’amico et al. 2003). In our study, the slope 246 of PSA biomarker was intended as a surrogate endpoint for PC recurrence. The PSA slope is 247 defined as the rate of PSA change over time following RT. For PSA slope calculation, there is a 248 controversy regarding the starting time and end of follow-up time. Anwar et al. (2014) calculated 
249 PSA slope as outcome after radiotherapy for 3 intervals following RT (0 to 1 year, 0 to 2 years, 250 and 0 to 3 years) and reported that the median PSA slope for conventionally-fractionated external 251 beam radiotherapy (CF-EBRT) was $-0.09,-0.04,-0.02 \mathrm{ng} / \mathrm{ml} / \mathrm{month}$, for durations of 1,2 and 3 252 years post RT. Similarly, for stereotactic body radiotherapy (SBRT), the median PSA slopes were $253-0.09,-0.06,-0.05 \mathrm{ng} / \mathrm{ml} / \mathrm{month}$. The PSA slope for SBRT was greater than CF-EBRT $(\mathrm{p}<0.05)$ 254 at 2 and 3 years following RT, although similar during the first year. PSA evolution following RT 255 was described by Prosto Lima et al with 3 components: post-therapy level, short-term decline 256 with exponential function of time and long-term PSA rise as a linear function of time. Takamiya 257 et al. (2003) calculated PSA slope for each patient with starting time 3 years after RT. 258 Antonarakis et al (2012) compared the pre-study entry (pre-treatment) PSA slope and post259 treatment PSA slope to describe the association between PSA kinetic and metastasis-free 260 survival. Although the connection between pre- and post- treatment PSA kinetic may clarify the role of PSA slope as a predictor or response variable in various studies. But in the retrospective studies, mostly the frequency of pre-treatment PSA evaluation is not regulated. Therefore, pretreatment PSA slope in majority of patients cannot be computed. For ease of applicability we included the last pre-treatment PSA value into serially PSA measurements after RT for each patient. With incorporating the pre-treatment PSA value into slope calculation directly a timedependent covariate to be joined to post-treatment PSA behavior. Also it takes into account the PSA change at during treatment and reduces the time dependent interval censoring bias.

While some studies have shown that there is a relationship between the magnitude of the PSA decline and clinical outcomes such as: development of distant metastases, risk for relapse (Kaplan, Cox \& Bagshaw 1991 and Chauvet et al. 1994) and metastasis- free survival (Antonarakis et al. 2012), other studies have not shown this relation (Ritter et al. 1992 and Zagars, Pollack 1993, 1997). In our study, with increasing dose level, the posterior mean of the PSA slope dropped with a faster rate in most patients (176 out of 195, $=90 \%$ ) and disease-free survival time in these patients was increased. This means that although there is better therapeutic response with higher doses of RT in prostate cancer, but the PSA slope changes as a mediate variable cannot fully save the dose effect on true response. Of course, this does not necessarily mean more benefit to the patient, because other endpoints such as early and late complications, metastasis-free survival and OS must be studied as well. 
281 and genitourinary toxicity complications also increased (Wang et al 2014, Zietman et al 2005, Al282 Mamgani et al. 2008 and Peeters et al. 2006). The initial studies were performed with a three 283 dimensional (3D) technique. Further studies showed that advanced methods of radiotherapies for 284 PC, such as Intensity modulated radiation treatment (IMRT), were associated with fewer side 285 effects, although they did not increase OS (Budäus et al. 2012, Wolff et al. 2009). In another 286 randomized dose escalation, in 301 patients were investigated in terms of doses of 78 Gy and 70 287 Gy. Pollack et al. showed that an increase of 8 Gy resulted in a highly significant improvement in 288 freedom from BF (70\% and 64\%, respectively $(\mathrm{p}=0.03)$ ) for patients at intermediate to high risk, 289 which is consistent with our study in freedom from recurrence (Pollack et al. 2002, Kuban et al. 2008). Overall survival improvement and advantage for dose escalation from 64.8 Gy to 86.4 Gy were reported in all risk groups (Zelefsky et al. 2011 and Hall et al. 2015) but Pollack et al.’s study (2004) has not demonstrated dose-escalation advantages for low-risk cases. Another study (Shelan et al. 2013) strongly supported the application of at least 70 Gy rather than 66 Gy. The results of our study revealed that however doses greater than 70 Gy decreased PSA more than other dose levels ( $<50$, [50 70), 70 Gy) but significantly increased disease-free survival time similar to above studies.

Another point about this study is that with using a simple approach, as linear regression, the last measurement before treatment and the pattern of PSA after treatment, were linked. Linear regression played the role of a flattening function. In fact, instead of a nonlinear function, the slope of the PSA as a smoothing function causes flexibility in the model. When PSA variability of within subjects is important and the true end points are related to complex biological process should not be relied on the values of PSA in the short term. Then long term follow up is needed for the PSA change point detection and two-component mixture model, exponential model and other approaches for tumor kinetic are suggested (Proust-Lim, et al. 2008).

In this paper, our focus was on the use of a potential surrogate biomarker for a Bayesian analysis of dose-response relationship. It was done by a retrospective review of the outcomes in prostate cancer patients. This feature limited the results of our study, so that our findings were restricted to patients with minimum time to clinical recurrence or lost to follow-up without recurrence as censored data. Short follow-up time will not allow us to determine the future disease progression or others true end points after recurrence. Since there is increasing interest in the use of surrogate marker endpoints in trials, further prospective clinical studies will be required in the field. 
312 Conclusion: Significant changes in the dose-response relationship were not observed when the 313 PSA slope was considered as the response criterion. Despite the fact that PSA slope was 314 prognostic factor for disease-free survival in this study, the association between PSA slope 315 changes and dose increase was not clear and therefore it cannot be used as a reliable surrogate for 316 PC recurrence endpoint.

\section{7}

\section{Acknowledgement:}

We thank all those who have helped us in collecting patient data, this article is part of a master's thesis Ms. Z. Faghani and supported by deputy of research in Mazandaran University of medical sciences.

\section{Data Deposition}

The raw data has been supplied as two Supplemental data set files: ProstateCancerDataS1.csv and an additional .xls EXCEL file contains the Trajectory PSA values "PSArepeatedMeasurementsS2". Data description (DatasetDescriptionS4) and R code has been supplied as supplemental information files.

\section{References:}

Antonarakis ES, Zahurak ML, Lin J, Keizman D, Carducci MA, \& Eisenberger MA. 2011. Changes in PSA kinetics predict metastasis- free survival in men with PSA-recurrent prostate cancer treated with non-hormonal agents: combined analysis of 4 phase II trials. Cancer 118:1533-42.

Alizadeh A, Mohammadpour RA, \& Barzegar MR. 2013. Comparing Cox Model and Parametric Models in Estimating the Survival Rate of Patients with Prostate Cancer on Radiation Therapy. Journal of Mazandaran University of Medical Sciences 23:21-29.

Al-Mamgani A, van Putten WL, Heemsbergen WD, van Leenders GJ, Slot A, Dielwart MF, Incrocci L. \& Lebesque JV. 2008. Update of Dutch multicenter dose-escalation trial of radiotherapy for localized prostate cancer. International Journal of Radiation Oncology• Biology• Physics 72:980-988.

Anwar M, Weinberg V, Chang AJ, Hsu I-Chow, Roach M. \& Gottschalk A. 2014. Hypofractionated SBRT versus conventionally fractionated EBRT for prostate cancer: comparison of PSA slope and nadir. Radiation Oncology 9:42. doi:10.1186/1748-717X-9-42.

Bellera CA, Hanley JA, Joseph L. \& Albertsen PC. 2008. Hierarchical changepoint models for biochemical markers illustrated by tracking postradiotherapy prostate-specific antigen series in men with prostate cancer. Annals of epidemiology 18: 4, 270-282.

Bidgoli SA, Jabari N \& Zavarhei MD. 2014. Prostate-specific antigen levels in relation to background factors: are there links to endocrine disrupting chemicals and AhR expression? APJCP 15: 6121-6125. 
344

345

346

347

348

349

350

351

352

353

354

355

356

357

358

359

360

361

362

363

364

365

366

367

368

369

370

371

372

373

374

375

376

377

378

379

380

381

382

383

Budäus L, Bolla M, Bossi A, Cozzarini C, Crook J, Widmark A. \& Wiegel T. 2012. Functional outcomes and complications following radiation therapy for prostate cancer: a critical analysis of the literature. European urology 61:112-127.

Chauvet B, Félix-Faure C, Lupsascka N, Fijuth J, Brewer Y, Davin J, Kirscher S \& Reboul F. 1994. Prostate-specific antigen decline: a major prognostic factor for prostate cancer treated with radiation therapy. Journal of clinical oncology 12: 1402-1407.

Collette L, Buyse M, Burzykowski T. 2007. Are prostate-specific antigen changes valid surrogates for survival in metastatic hormone-refractory prostate cancer? J Clin Oncol 25:5673-5674.

D'Amico AV, Moul JW, Carroll PR, Sun L, Lubeck D. \& Chen MH. 2003. Surrogate end point for prostate cancer-specific mortality after radical prostatectomy or radiation therapy. Journal of the National Cancer Institute 95:1376-1383.

D'Amico AV, Whittington R, Malkowicz SB, Schultz D, Blank K, Broderick GA, Tomaszewski JE, Renshaw AA, Kaplan I, Beard CJ, \& Wein A. 1998. Biochemical outcome after radical prostatectomy, external beam radiation therapy, or interstitial radiation therapy for clinically localized prostate cancer. JAMA 280: 969-974.

Esfahani M, Ataei N. \& Panjehpour M. 2014. Biomarkers for evaluation of prostate cancer prognosis. APJCP 16:2601-2611.

Halabi S, Armstrong AJ, Sartor O, de Bono J, Kaplan E, Lin CY, Solomon NC \& Small EJ. 2013. ProstateSpecific Antigen Changes As Surrogate for Overall Survival in Men With Metastatic CastrationResistant Prostate Cancer Treated With Second-Line Chemotherapy. Journal of Clinical Oncology 31:3944-3950.

Hall MD, Schultheiss TE, Smith DD, Tseng BP, \& Wong JYC. 2015. The impact of increasing dose on overall survival in prostate cancer. Radiation Oncology 10:115, DOI 10.1186/s13014-015-0419-3.

Hamra G, MacLehose R, \& Richardson D. 2013. Markov chain Monte Carlo: an introduction for epidemiologists. Int J Epidemiol 42:627-634.

Heller G. 2015. Statistical cotroversies in clinical research: an initial evaluation of a surrogate end point using a single randomized clinical trial and the Prentice criteria. Annals of Oncology 26:20122016.

Kaplan ID, Cox RS. \& Bagshaw MA. 1991. A model of prostatic carcinoma tumor kinetics based on prostate specific antigen levels after radiation therapy. Cancer 68:400-405.

Kuban DA, Tucker SL, Dong L, Starkschall G, Huang EH, Cheung MR, Lee AK, \& Pollack A. 2008. Longterm results of the M. D. Anderson randomized dose-escalation trial for prostate cancer. Int J Radiat Oncol Biol Phys 70:67-74

Leininger TJ. 2009. An Adaptive Bayesian Approach to Continuous Dose-Response Modeling: Brigham Young University.

Molenberghs G, Buyse M, Geys H, Renard D, Burzykowski T. \& Alonso A. 2002. Statistical challenges in the evaluation of surrogate endpoints in randomized trials. Controlled clinical trials 23: 607-625.

Ntzoufras I. 2011. Bayesian modeling using WinBUGS: John Wiley \& Sons.

Obort AS, Ajadi MB. \& Akinloye O. 2013. Prostate-specific antigen: any successor in sight? Reviews in urology 15:3.

Peer] reviewing PDF | (2018:02:24790:3:1:NEW 22 May 2019) 
384

385

386

387

388

389

390

391

392

393

394

395

396

397

398

399

400

401

402

403

404

405

406

407

408

409

410

411

412

413

414

415

416

417

418

419

420

421

422

423

424

Peeters STH, Heemsbergen WD, Koper PCM, van Putten VLJ, Slot A, Dielwart MFH, Bonfrer JMG, Incrocci L \& Lebesque JV. 2006. Dose-Response in Radiotherapy for Localized Prostate Cancer: Results of the Dutch Multicenter Randomized Phase III Trial Comparing 68 Gy of Radiotherapy With $78 \mathrm{~Gy}$. Journal of clinical oncology 24:13.

Pollack A, Hanlon AL, Horwitz EM, Feigenberg SJ, Uzzo RG and Hanks GE. 2004. Prostate cancer radiotherapy dose response: an update of the Fox Chase experience. J Urol 171:1132-1136

Pollack A, Zagars GK, Starkschall G, Antolak JA, Lee JJ, Huang E, von Eschenbach AC, Kuban DA. \& Rosen I. 2002. Prostate cancer radiation dose response: results of the M. D. Anderson phase III randomized trial. International Journal of Radiation Oncology Biol. Phys 52:1097-1105 .

Prentice RL. 1989. Surrogate endpoints in clinical trials, definition and operational criteria. Statistics in Medicine 8:431-440.

Proust-Lima C, Taylor JMG, Williams SG, Ankerst DP, Liu N, Kestin LL, Bae K \& Sandler HM. 2008. Determinants of change in prostate-specific antigen over time and its association with recurrence after external beam radiation therapy for prostate cancer in five large cohorts. Int J Radiat Oncol Biol Phys 72:782-791.

Ritter MA, Messing EM, Shanahan TG, Potts S, Chappell RJ, Kinsella TJ. 1992. Prostate-specific antigen as a predictor of radiotherapy response and patterns of failure in localized prostate cancer. Journal of clinical oncology 10: 1208-1217.

Rodrigues G, Warde P, Pickles T, Crook J, Brundage M, Souhami L, \& Lukka H. 2012. Pre-treatment risk stratification of prostate cancer patients: A critical review. Canadian Urological Association Journal 6:2.

Shelan M, Abo-Madyan Y, Welzel G, Bolenz C, Kosakowski J, Behnam N, Wenz F. \& Lohr F. 2013. Dose-escalated salvage radiotherapy after radical prostatectomy in high risk prostate cancer patients without hormone therapy: outcome, prognostic factors and late toxicity. Radiation Oncology 8:276. doi:10.1186/1748-717X-8-276.

Smith BJ. 2007. Boa: an R package for MCMC output convergence assessment and posterior inference. Journal of Statistical Software 21:1-37.

Suzman DL, Zhou XC, Zahurak ML, Lin J. \& Antonarakis ES. 2015. Change in PSA velocity is a predictor of overall survival in men with biochemically-recurrent prostate cancer treated with nonhormonal agents: combined analysis of four phase-2 trials. Prostate cancer and prostatic diseases 18:49-55.

Taylor JM, \& Wang Y. 2002. Surrogate markers and joint models for longitudinal and survival data. Controlled Clinical Trials 23:626-634.

Takamiya R, Weinberg V, Young CD, Sandler H, McLaughlin P, \& Roach lii M. 2003. A zero PSA slope in posttreatment prostate-specific antigen supports cure of patients with long-term follow-up after external beam radiotherapy for localized prostate cancer. Int J Radiat Oncol Biol Phys 56:1073-1078.

Valicenti RK, DeSilvio M, Hanks GE, Porter A, Brereton H, Rosenthal S, Shipley WU, \& Sandler HM. 2006. Post treatment prostatic-specific antigen doubling time as a surrogate endpoint for prostate cancer-specific survival: an analysis of Radiation Therapy Oncology Group Protocol 92-02. Int J Radiat Oncol Biol Phys 66:1064-1071.

Vollmer RT. \& Montana GS. 1999. The dynamics of prostate-specific antigen after definitive radiation therapy for prostate cancer. Clinical cancer research 5: 4119-4125. 
425

426

427

428

429

430

431

432

433

434

435

436

437

438

439

440

441

442

443

444

445

446

447

448

449

450

451

452

453

454

455

456

457

458

459

460

461

462

463

Wang D, Ho H, Hamilton AS, Wu Xiao-Cheng Lo M, Fleming S, Goodman M, Thompson T. \& Owen J. 2014. Type and dose of radiotherapy used for initial treatment of non-metastatic prostate cancer. Radiation Oncology 9:47. doi:10.1186/1748-717X-9-47.

Wilt TJ. \& Ahmed HU. 2013. Prostate cancer screening and the management of clinically localized disease. BMJ 346: $\mathrm{f} 325$.

Wolff D, Stieler F, Welzel G, Lorenz F, Abo-Madyan Y, Mai S, Herskind C, Polednik M, Steil V, Wenz F. \& Lohr F. 2009. Volumetric modulated arc therapy (VMAT) vs. serial tomotherapy, step-and-shoot IMRT and 3D-conformal RT for treatment of prostate cancer. Radiotherapy and oncology 93:226-233.

Zagars GK. \& Pollack A. 1993. The fall and rise of prostate-specific antigen: Kinetics of serum prostate-specific antigen levels after radiation therapy for prostate cancer. Cancer 72: 832-842.

Zagars GK. \& Pollack A. 1997. Kinetics of serum prostate-specific antigen after external beam radiation for clinically localized prostate cancer. Radiotherapy and oncology 44:213-221.

Zelefsky MJ, Pei X, Chou JF, Schechter M, Kollmeier M, Cox B, Yamada Y, Fidaleo A, Sperling D, Happersell L, \& Zhang Z. 2011. Dose escalation for prostate cancer radiotherapy: predictors of longterm biochemical tumor control and distant metastases-free survival outcomes. Eur Urol 60:11331139

Zietman AL, DeSilvio ML, Slater JD, Rossi CJ Jr, Miller DW, Adams JA \& Shipley WU. 2005. Comparison of conventional-dose vs high-dose conformal radiation therapy in clinically localized adenocarcinoma of the prostate: a randomized controlled trial. JAMA 294:1233-1239.

Table 1 Frequency distribution (\%) of prostate cancer patients in the non-stratified and stratified based on risk and radiation dose level

Table 2 Posterior estimations of PSA slope means in a Bayesian model; without stratification

Figure 1 Scatter plot for correlation between follow-up period and PSA slope after RT

Figure 2 (A-D) Boxplots for comparison of the dose level posterior means in the non-stratified and stratified patients

Figure 3(A, B) Disease-free survival function by (A) total dose levels and (B) PSA slope after RT

Figure 4(A, B) Disease-free survival function by (A) total dose levels in PSA slope $<=0$, (B) total dose levels in PSA slope $>0$ 
464

465 
Figure 1

Scatter plot for correlation between follow-up period and PSA slope after RT

Slope of PSA after radiotherapy $(\mathrm{ng} / \mathrm{ml} / \mathrm{month})$

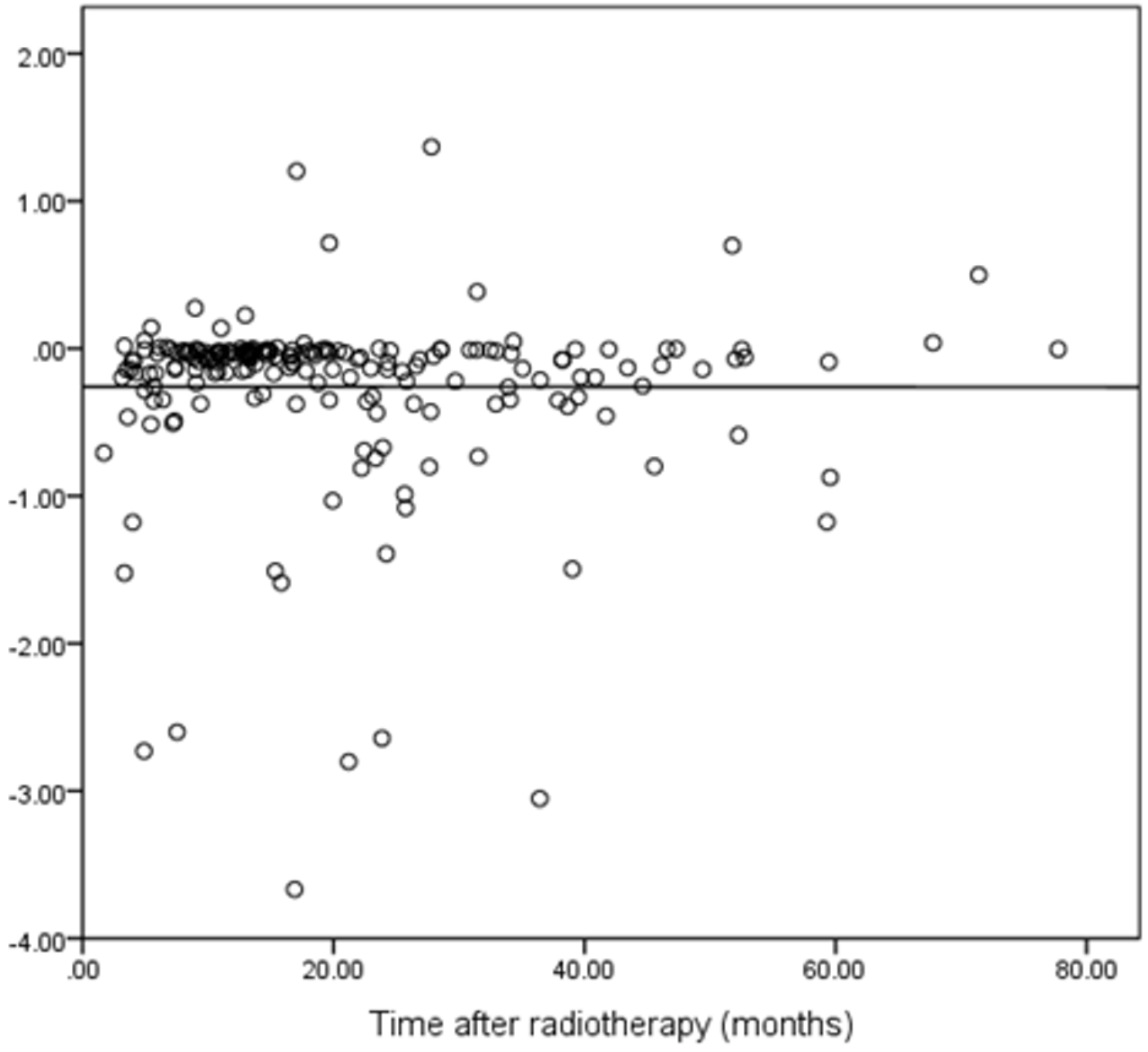


Figure 2

(A-D) Boxplots for comparison of the dose level posterior means in the non-stratified and stratified patients.
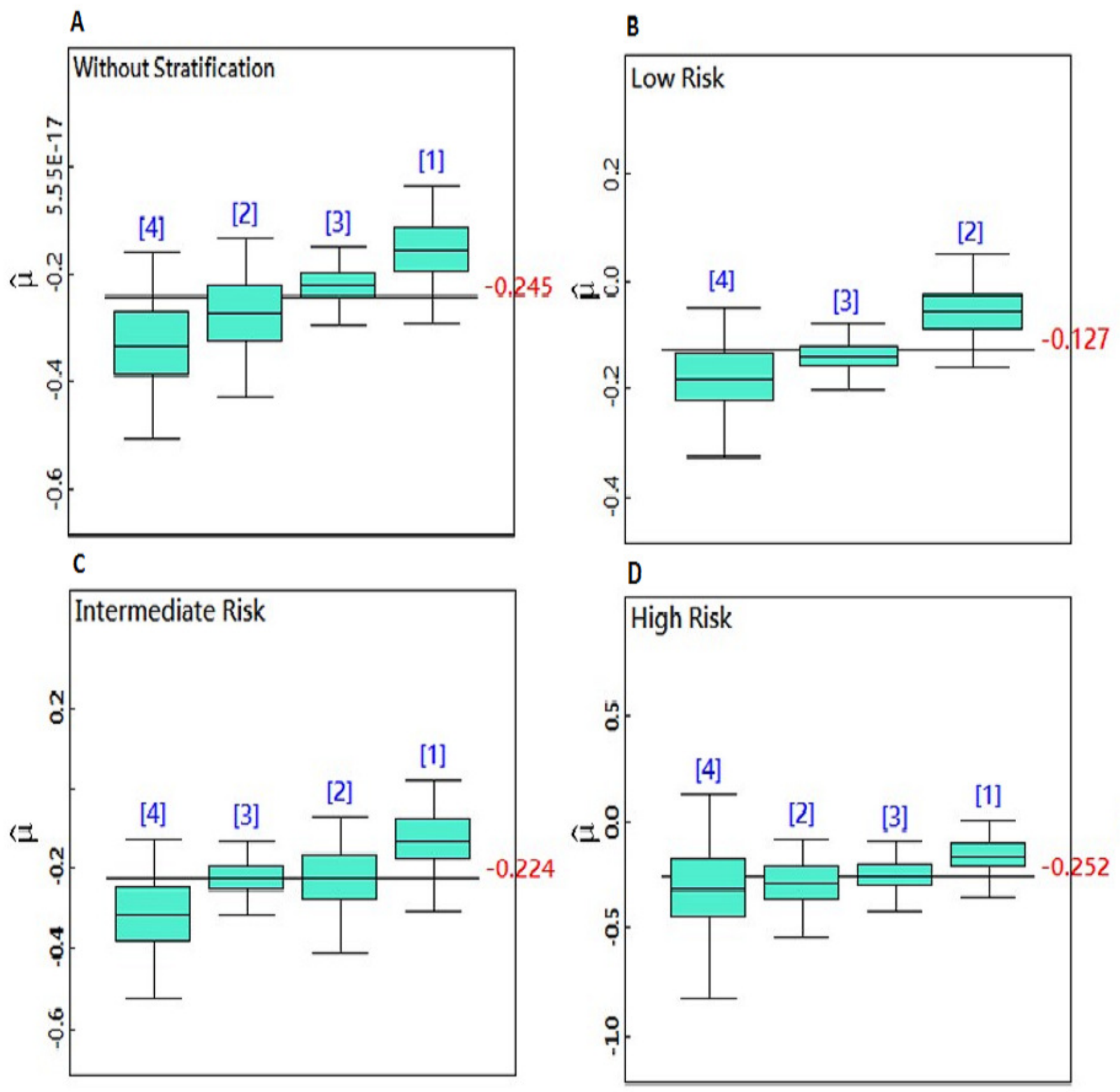
Figure 3

(A, B) Disease-free survival function by (A) total dose levels and (B) PSA slope after RT 

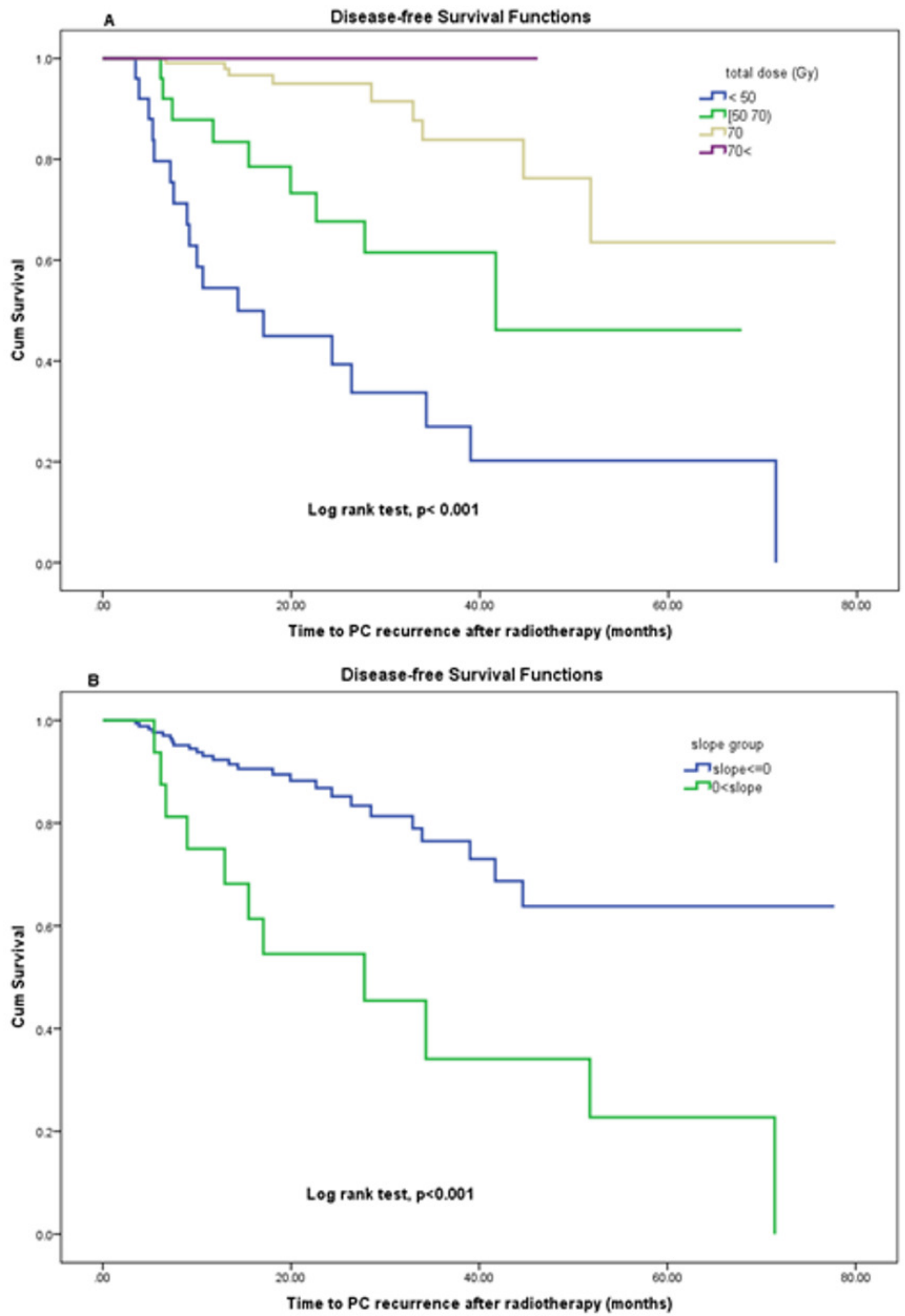

Peer] reviewing PDF | (2018:02:24790:3:1:NEW 22 May 2019) 
Figure 4

(A, B) Disease-free survival function by $(A)$ total dose levels in PSA slope $<=0$, (B) total dose levels in PSA slope $>0$ 


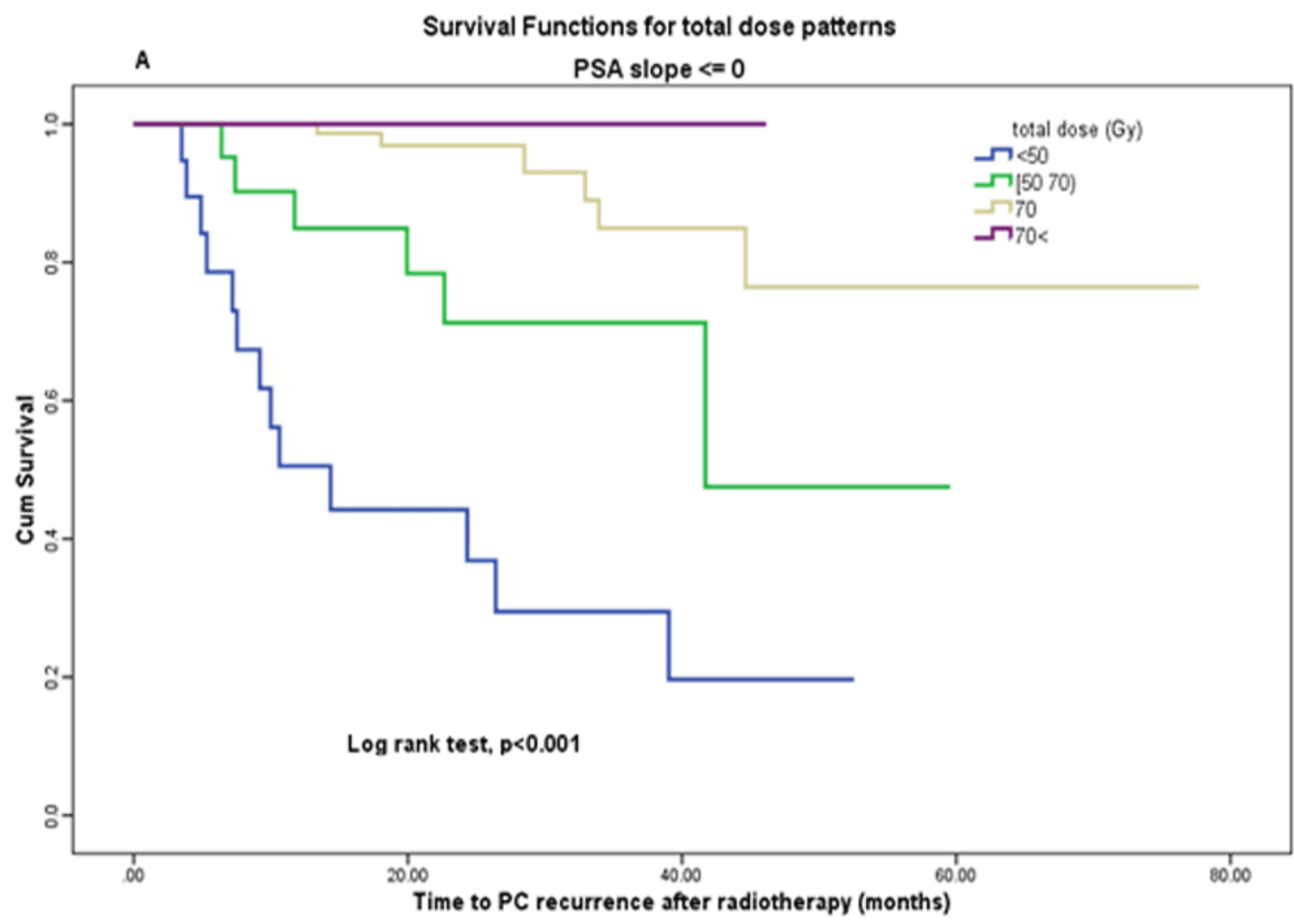

B

Survival Functions for total dose patterns

PSA slope $>0$

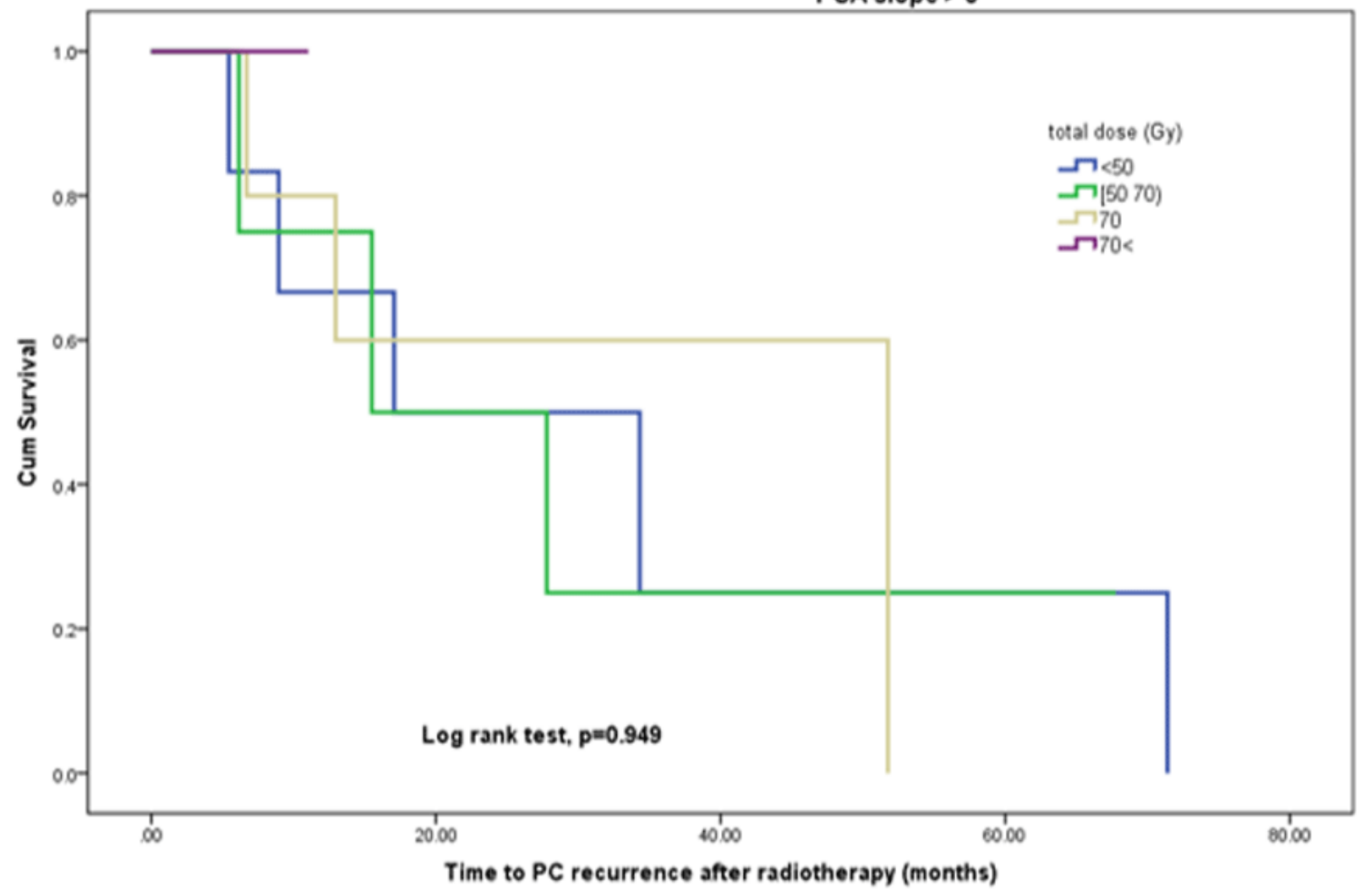




\section{Table $\mathbf{1}$ (on next page)}

Frequency distribution (\%) of prostate cancer patients in the non-stratified and stratified based on risk and radiation dose level 
1

\begin{tabular}{|c|c|c|c|c|c|}
\hline Groups & $<50$ Gy & {$[50$ 70) Gy } & $=70 \mathrm{~Gy}$ & $70<\mathbf{G y}$ & Total \\
\hline $\begin{array}{c}\text { Without } \\
\text { Stratification }\end{array}$ & $24(12.31)$ & 30 (15.38) & $119(61.03)$ & $22(11.28)$ & 195 \\
\hline Low Risk & - & $3(11.54)$ & $20(76.92)$ & 3 (11.54) & $26(13.34)$ \\
\hline $\begin{array}{c}\text { Intermediate } \\
\text { Risk }\end{array}$ & $8(7.92)$ & 15 (14.85) & $64(63.37)$ & $14(13.86)$ & 101 (51.79) \\
\hline High Risk & $16(23.53)$ & 12 (17.65) & 35 (51.47) & $5(7.35)$ & $68(34.87)$ \\
\hline
\end{tabular}

2 


\section{Table 2 (on next page)}

Posterior estimations of PSA slope means in a Bayesian model; without stratification 


\begin{tabular}{|c|c|c|c|c|c|c|}
\hline Dose level & mean & $\begin{array}{c}\text { Standard } \\
\text { deviation }\end{array}$ & $\begin{array}{c}2.5 \% \\
\text { percentiles }\end{array}$ & Median & $\begin{array}{c}97.5 \% \\
\text { percentile }\end{array}$ & $\begin{array}{c}\text { Gelman- } \\
\text { Rubin } \\
\text { statistic }\end{array}$ \\
\hline$<\mathbf{5 0}$ Gy & -0.1543 & 0.0654 & -0.2909 & -0.1509 & -0.0323 & 1.0005 \\
\hline$[\mathbf{5 0} \mathbf{7 0}) \boldsymbol{G} \boldsymbol{y}$ & -0.2733 & 0.0775 & -0.4278 & -0.2723 & -0.1275 & 1.0013 \\
\hline $\mathbf{7 0} \mathbf{G} \boldsymbol{y}$ & -0.2203 & 0.0379 & -0.2952 & -0.2201 & -0.1464 & 1.0001 \\
\hline $\mathbf{7 0}<\mathbf{G y}$ & -0.332 & 0.0903 & -0.5086 & -0.3319 & -0.1537 & 1.0003 \\
\hline
\end{tabular}

